\title{
A Literature Review of Pregnancy Worries and Stress Scales
}

\author{
Fatemeh Abdi $^{1}$, Fariba Navidpour ${ }^{2}$ and Mahrokh Dolatian ${ }^{2,{ }^{*}}$ \\ ${ }^{1}$ Student Research Committee, Faculty of Nursing and Midwifery, Shahid Beheshti University of Medical Sciences, Tehran, Iran \\ ${ }^{2}$ Department of Midwifery and Reproductive Health, Faculty of Nursing and Midwifery, Shahid Beheshti University of Medical Sciences, Tehran, Iran \\ "Corresponding author: Mahrokh Dolatian, Assistant Professor, Department of Reproductive Health and Midwifery, School of Nursing and Midwifery, Shahid Beheshti \\ University of Medical Sciences, Tehran, Iran. Tel: +98-2188202517, Fax: +98-2188202520, Email: mhdolatian@gmail.com
}

Received 2017 June 11; Revised 2018 February 05; Accepted 2018 April 13.

\begin{abstract}
Context: High levels of worries and stress in pregnancy result in negative pregnancy outcomes and postnatal depression. Despite recommendations for routine psychological evaluation of pregnant women, there is a lack of scales specifically designed to measure stress during pregnancy.

Evidence Acquisition: PubMed, Medline, Scopus, ISI Web of Knowledge, ScienceDirect, PsycINFO, and Google Scholar databases were searched for English articles published during 1983 - 2016. The consensus-based standards for the selection of health status measurement instruments (COSMIN) checklist was used for assessing the methodological quality of the studies.

Results: A total of 27 studies were included, and 27 different instruments were identified. Validity and reliability assessments were performed in all the papers. According to the COSMIN checklist, the overall quality of the papers was fair to excellent. Among all the instruments, the pregnancy worries and stress questionnaire (PWSQ) and pregnancy-related anxiety questionnaire (PRAQ) showed moderate to strong evidence in most of the evaluated measurement properties.

Conclusions: A few scales with acceptable theoretical and psychometric properties are available for the measurement of pregnancy worries. General tools previously adopted to assess worries and stress in pregnancy need further psychometric testing to confirm their reliability and suitability for the prenatal period. The results of this study indicated that while none of the evaluated tools was completely suitable for the assessment of worries and stress in pregnancy, the PWSQ and PRAQ showed moderate to strong evidence in most of the examined measurement properties.
\end{abstract}

Keywords: Pregnancy, Anxiety, Worries, Pregnancy Worries Scale, Pregnancy Anxiety Scale

\section{Context}

Pregnancy worries are defined as a specific type of psychological distress experienced by a pregnant woman regarding her health, her baby's health, her body image, and delivery (1). Various biological and environmental conditions, along with changes in personal relationships, cause high levels of stress and anxiety in pregnant women (2). Concerns about the newborn's health, pregnancy complications, pregnancy loss, and childbirth are the common sources of worries in pregnant women. Also, external stressors such as worries about money, job, housing, health, and marital relationships can interfere with the natural course of pregnancy (3).

The prevalence of prenatal depression varies from $7 \%$ - $20 \%$ in high-income countries to over $20 \%$ in middleand low-income countries (4). Meanwhile, $16.7 \%-74 \%$ of women suffer from prenatal tension and anxiety during the first trimester of pregnancy (5).

While pregnancy anxiety is a strong predictor of ad- verse birth and infant outcomes, the exact role of different sources of prenatal stress, depression, anxiety, and pregnancy-specific anxiety in infants' emotional reactivity is still unclear (6). A previous study reported a relationship between prenatal anxiety and high negative emotionality in infants who received low postnatal stroking (7). A recent review identified antenatal worries and anxiety, along with a previous history of psychiatric illnesses, poor marital relationship, stressful life events, negative attitude toward pregnancy, and lack of social support, as significant risk factors for postnatal depression (8). It is, hence, essential to determine the extent and causes of pregnancy worries and anxiety.

While a national prenatal screening program for anxiety and depression is performed to measure pregnant women's vulnerability in Australia, such formal screening procedures are not as widely used in the United States or United Kingdom (9). The National Institute for Health and Care Excellence and the Healthy Child Program in England have both emphasized the necessity of determining the 
effects of prenatal anxiety on women and their offspring (10). Despite the high prevalence of pregnancy worries and stress, their assessment using suitable scales has not been widely investigated in the Middle Eastern countries. This highlights the need for the evaluation of the available scales for pregnancy worries and stress.

Various tools and measures, including the state-trait anxiety inventory (STAI) (11) and Cambridge worry scale (CWS) (12), have been administered to examine the level of prenatal tension, stress, and worries. However, the use of these tools is associated with several problems. Most importantly, while these scales evaluate general anxiety and worries, a pregnant woman, who is not depressed and anxious due to other reasons, might be concerned about both childbirth and her unborn baby's health (13). The identification and measurement of pregnancy worries would thus require a valid and reliable scale specifically designed for pregnant women. Systematic reviews are powerful tools to summarize the existing research and provide a large body of evidence about a particular subject of interest (14). However, to the best of our knowledge, there are shortcomings in previous reviews of the available instruments for pregnancy worries and stress. In fact, although the existing reviews have presented significant information in this field, they present contradictory information. Brunton et al. reviewed the scales used to assess pregnancy-related anxiety, and Alderdice et al. provided a psychometric analysis of the existing pregnancy-specific maternal stress measures. These reviews differ from this study by construct ( 9 , 15). Therefore, this article aimed to review the literature regarding pregnancy worries scales.

\section{Evidence Acquisition}

\subsection{Search Strategy}

In order to find the relevant studies, we searched the Cochrane database of systematic reviews (via Cochrane library), PubMed, Medline, Scopus, ISI Web of Knowledge, ScienceDirect, Psyc INFO, and Google Scholar. We used the following keywords (Mesh-terms): "pregnancy" AND ("stress" OR "anxiety" OR "worries"), "pregnancy worries scale" OR "pregnancy stress scale" OR "pregnancy anxiety scale" OR "prenatal stress scale" OR "pregnancy-related anxiety scale." Articles were included if they were published in English during 1983 - 2016 and described an instrument to measure or screen pregnancy-related worries, stress, and anxiety.

\subsection{Inclusion Criteria}

Studies that used an existing instrument or developed a particular tool to investigate the presence of pregnancyrelated worries and stress and/or measure their intensity were included. A wide range of studies, including published theses in Persian language, were selected.

\subsection{Exclusion Criteria}

We excluded articles on a topic unrelated to our review or those that did not specifically focus on pregnancyrelated worries, distress, anxiety, and depression. Also, studies which lacked numerical outcome data were not selected.

\subsection{Study Selection}

Two authors selected the eligible studies and resolved cases of disagreement through consensus. Overall, one thesis and 4870 papers were individually assessed, and the duplicates were excluded. Following the evaluation of the abstracts and titles of the remaining 2360 papers, 2319 irrelevant articles were excluded, and the full texts of the remaining 41 articles were examined. While 27 papers were found eligible, 14 papers were excluded since they did not provide specific information about the administered scales. The flow diagram of the literature review process is presented in Figure 1.

\subsection{Quality Assessment}

The consensus-based standards for the selection of health status measurement instruments (COSMIN) checklist with a four-point rating scale was used for the quality assessment of the selected papers. COSMIN is a standardized tool for assessing the methodological quality of studies on measurement properties of health measurement instruments. The checklist consists of nine boxes to evaluate nine different measurement properties including internal consistency, reliability, measurement error, content validity, structural validity, hypothesis testing, cross-cultural validity, criterion validity, and responsiveness (16). Each box contains 5 - 18 items about the applied design and statistical methods.

\subsection{Data Extraction}

The selected articles were carefully reviewed, and the required data, including the author names and publication year, the name of the administered instrument, items and response format of the instrument, data about reliability and validity, and description of the instrument, were extracted and reported. 


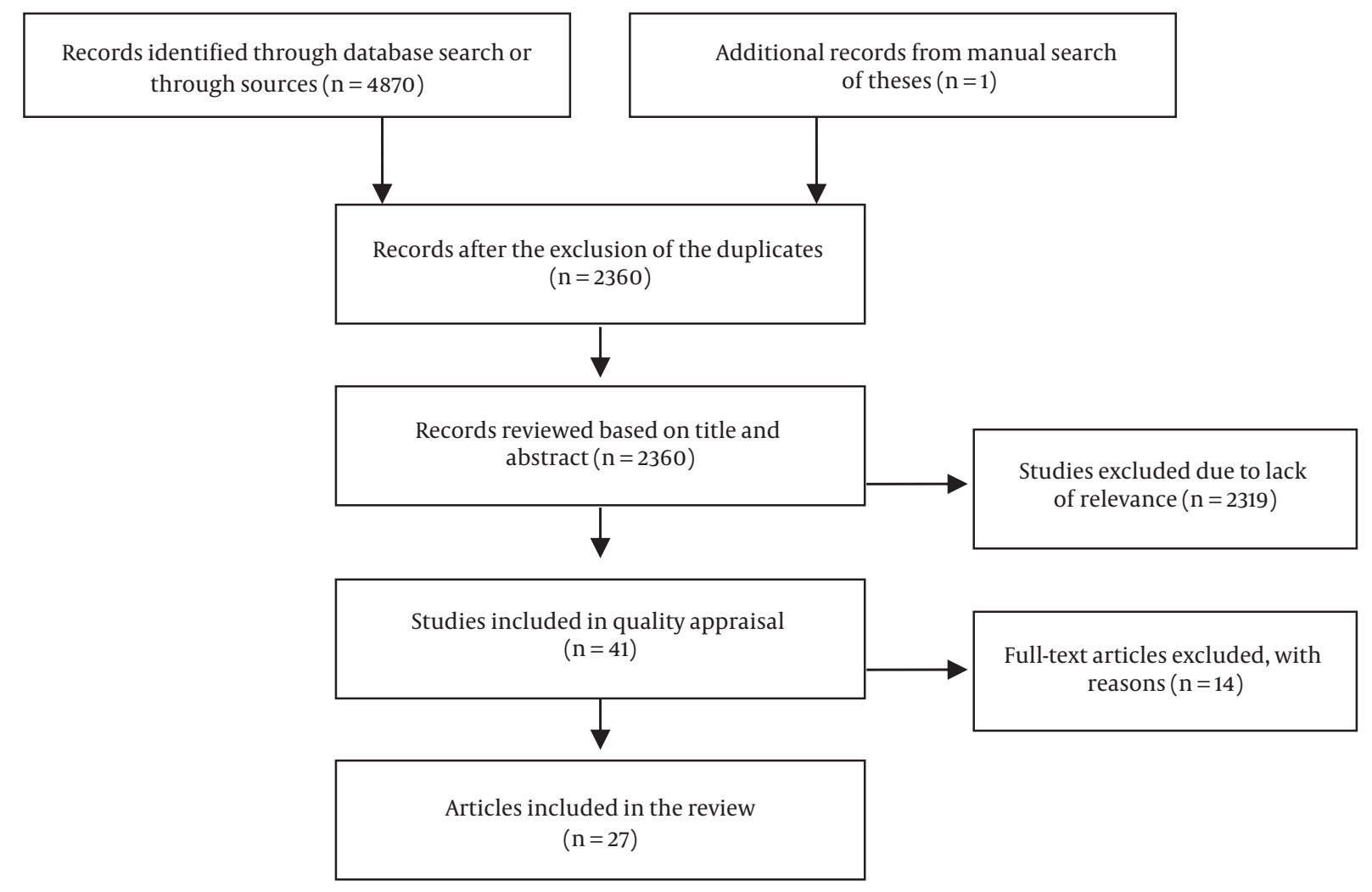

Figure 1. Search flow diagram

\section{Results}

A total of 27 different scales were used in the 27 selected studies (Table 1). While all the studies reported reliability data, test-retest reliability was calculated by 10 (37\%) studies. More than one type of validity was discussed in most articles. Content, criterion, convergent, construct, discriminate, concurrent, and face validity were reported by three, five, two, seven, one, five, and three studies, respectively. Hypothesis testing was performed in nine studies, and factor structure was evaluated in four papers. Of the 27 identified scales, eight instruments, including the pregnancy-related anxiety questionnaire (PRAQ), pregnancy-related anxiety questionnaire-revised (PRAQ-R), pregnancy-related anxiety questionnaire short (PRAQ-S), pregnancy anxiety scale (PAS), pregnancy-specific anxiety scale (PSAS), and pregnancy-specific anxiety scale-revised (PSAS-R), specifically focused on pregnancy-related anxiety. Since two scales shared the name of the PAS and two shared the name of the PSAS, scale names were prefixed with author names for clarity. Moreover, two general anxiety scales, that is, STAI and Penn State Worry questionnaire, were included in this review.
The selected studies evaluated a wide range of instruments including tools specifically designed to measure pregnancy-related worries and stress. These tools included pregnancy worries and stress questionnaire (PWSQ), pregnancy-related thoughts, prenatal social environment inventory, baby schema questionnaire, Tilburg pregnancy distress scale, rural pregnancy experience scale, A-Z stress scale, CWS, Cambridge worry scale-revised (CWS$\mathrm{R})$, high risk pregnancy stress, Oxford worries about labor scale, pregnancy outcome questionnaire, pregnancy experience scale (PES), pregnancy experience scale-revised (PES-R), pregnancy experience questionnaire, prenatal psychosocial profile stress, and prenatal distress questionnaire. Some of the mentioned tools, including the PRAQ-R, PRAQ-S, CWS-R, PSAS-R, and PES-R, were the subscales of a longer instrument.

\section{Discussion}

This systematic review focused on instruments evaluating pregnancy-related worries and stress in the domains of health promotion and maintenance. While none of 


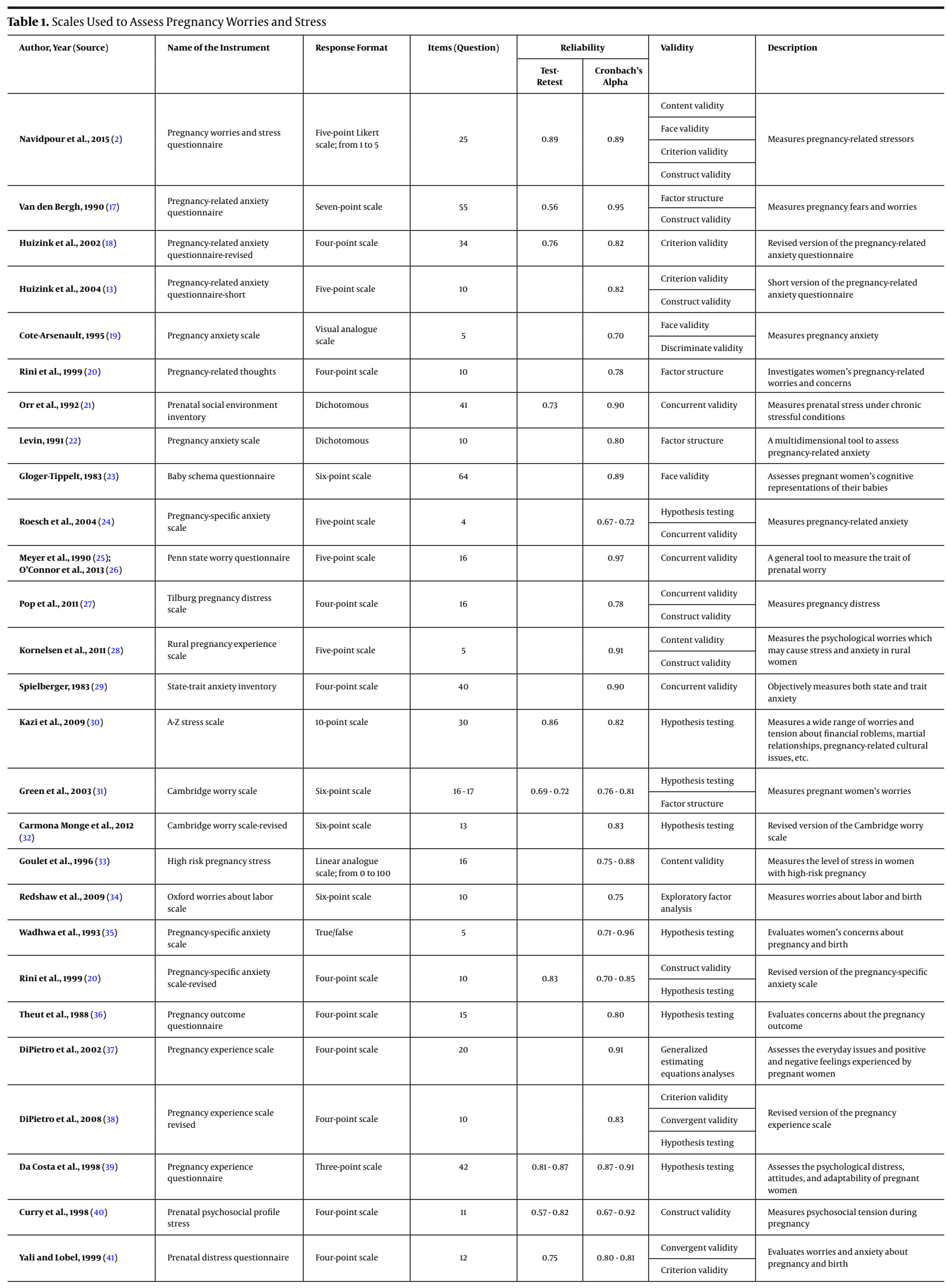


the evaluated instruments were completely suitable for the assessment of worries and stress in healthy pregnant women, the PWSQ, PRAQ, and PRAQ-R showed moderate to strong evidence in most of the examined measurement properties. However, when selecting a particular scale to measure pregnancy worries, the clinicians, nurses, and researchers need to consider not only the characteristics of the target population and settings, but also several factors related to the scale (e.g., the number of items and the specific contents or dimensions of the scale). Pregnancyrelated stress consists of fears and worries specifically experienced by pregnant women $(13,42)$. For instance, the PRAQ (17) does not have adequate items to identify the general symptoms commonly experienced by pregnant women. Meanwhile, the scopes of Levin's PAS, PRAQ-R, and PRAQ-S are not wide enough, and they are not suitable for evaluating the aspect of anxiety.

Psychometric evaluations, including validity and reliability testing, are also essential when selecting a scale (43). Among the 27 studied scales, the PWSQ (2) enjoyed good validity and reliability, and its items were not ambiguous for the respondents. Nevertheless, its psychometric properties should be further investigated. This scale can be validated and widely used. This assessment is based on correlations with the STAI in a sample of 100 pregnant women. However, this scale does not have a special cut off score, that is, this scale identifies stressors. Despite its good reliability, Levin's PAS was not widely administered, probably due to the limitations caused by its dichotomous responses. No data about the validation of this scale was provided in the selected studies. The PRAQ, developed by Van den Berg, had the greatest factor loading. The scale was later shortened due to low factor loading, and some items were excluded due to high error variance. The PRAQ-R and PRAQ-S could not be independently used as they had limited scopes and failed to consider the physiological aspect of anxiety. In fact, none of the studied pregnancy-specific scales considered the physiological aspect of anxiety. The pregnancy-related thoughts covered a wide range of fears and worries but included only a single indicator for each. Shorter scales assess pregnancy-related worries through one item. Despite their advantages, the limited number of items in shorter scales usually results in poorer psychometric properties (43). On the other hand, as indicated by some researchers, a large number of items might prevent the respondents from providing accurate responses (44). Although some of the studied scales could predict pregnancy outcomes, they sometimes lack a scientific background (15).

Moreover, some scales did not yield different results when measuring pregnancy-related worries and anxiety in general. Therefore, pregnancy-related scales developed based on the relevant scientific principles can be more reliably used for the identification of pregnancy-specific worries. This will, in turn, facilitate the design of effective interventions to decrease the stress and worries experienced by pregnant women. Furthermore, successful health assessment in pregnant women will depend on the integrated use of well-designed tools. Considering the negative effects of pregnancy-related worries and stress on maternal and fetal health, developing a valid, reliable, and usable scale for the identification of sources of stress in pregnant women seems critical. Relevant interventions can then be designed based on the information obtained from the administration of such a scale.

Finally, some items of particular scales can be confounding for pregnant women. Moreover, incompatible results might be obtained if a scale contains highly somatic contents or ambiguous items such as nausea, vomiting, and breathlessness, common symptoms experienced by many pregnant women (45).

\subsection{Limitations}

Like any other study, this systematic review has some limitations. Some studies had low methodological quality as their sample size was too limited for the number of items included in the scale. In addition, the evaluation of the methodological quality of the studies and the quality of the results might have been affected by the information on measurement properties presented in the reviewed articles.

\subsection{Conclusion}

Pregnancy-related stress is a definite anxiety response in pregnant women. Since stress and worries can cause negative pregnancy outcomes including preterm labor and low birth weight infants, a well-developed scale with acceptable performance and psychometric properties is required to measure pregnancy-related worries. General tools previously adopted to assess worries and stress in pregnancy need further psychometric testing to confirm their reliability and suitability for the prenatal period. A well-developed instrument is of high clinical significance as it can facilitate the design of relevant interventions to decrease stress and anxiety in pregnant women. Further research toward the development of such scales is warranted.

\section{Acknowledgments}

We are grateful for the helpful comments of anonymous referees. 


\section{Footnotes}

Authors' Contribution: Fatemeh Abdi and Mahrokh Dolatian conceived the study, interpreted the data, and drafted the manuscript. Fariba Navidpour acquired the data and interpreted the data. All the authors approved the final version of the paper.

Declaration of Interests: None.

Funding/Support: Shahid Beheshti University of Medical Sciences, Tehran, Iran.

\section{References}

1. Kane HS, Dunkel Schetter C, Glynn LM, Hobel CJ, Sandman CA Pregnancy anxiety and prenatal cortisol trajectories. Biol Psychol. 2014;100:13-9. doi: 10.1016/j.biopsycho.2014.04.003. [PubMed: 24769094]. [PubMed Central: PMC4170592]

2. Navidpour F, Dolatian M, Yaghmaei F, Majd HA, Hashemi SS. Examining factor structure and validating the Persian version of the pregnancy's worries and stress questionnaire for pregnant Iranian women. Glob J Health Sci. 2015;7(6):308-18. doi: 10.5539/gjhs.v7n6p308. [PubMed: 26153186]. [PubMed Central: PMC4803912].

3. Mortazavi F, Akaberi A. Worries of pregnant women: Testing the Farsi Cambridge worry scale. Scientifica (Cairo). 2016;2016:5791560. doi: 10.1155/2016/5791560. [PubMed: 27293974]. [PubMed Central: PMC4886057].

4. Biaggi A, Conroy S, Pawlby S, Pariante CM. Identifying the women at risk of antenatal anxiety and depression: A systematic review. J Affect Disord. 2016;191:62-77. doi: 10.1016/j.jad.2015.11.014. [PubMed: 26650969]. [PubMed Central: PMC4879174].

5. Navidpour F, Dolatian M, Shishehgar S, Yaghmaei F, Majd HA Hashemi SS. Validating the Farsi version of the pregnancy worries and stress questionnaire (PWSQ): An exploratory factor analysis. Electron Physician. 2016;8(10):3132-7. doi: 10.19082/3132. [PubMed: 27957315]. [PubMed Central: PMC5133040].

6. Nolvi S, Karlsson L, Bridgett DJ, Korja R, Huizink AC, Kataja EL, et al. Maternal prenatal stress and infant emotional reactivity six months postpartum. J Affect Disord. 2016;199:163-70. doi: 10.1016/j.jad.2016.04.020. [PubMed: 27107891].

7. Sharp H, Hill J, Hellier J, Pickles A. Maternal antenatal anxiety, postnatal stroking and emotional problems in children: Outcomes predicted from pre- and postnatal programming hypotheses. Psychol Med.2015;45(2):269-83. doi:10.1017/S0033291714001342.[PubMed: 25068652]. [PubMed Central: PMC4301199].

8. Norhayati MN, Hazlina NH, Asrenee AR, Emilin WM. Magnitude and risk factors for postpartum symptoms: A literature review. J Affect Disord. 2015;175:34-52. doi:10.1016/j.jad.2014.12.041. [PubMed: 25590764].

9. Brunton RJ, Dryer R, Saliba A, Kohlhoff J. Pregnancy anxiety: A systematic review of current scales. J Affect Disord. 2015;176:24-34. doi: 10.1016/j.jad.2015.01.039. [PubMed: 25687280].

10. Evans K, Spiby H, Morrell CJ. A psychometric systematic review of self-report instruments to identify anxiety in pregnancy. J Adv Nurs. 2015;71(9):1986-2001. doi:10.1111/jan.12649. [PubMed: 25818179].

11. Alipour Z, Lamyian M, Hajizadeh E. Anxiety and fear of childbirth as predictors of postnatal depression in nulliparous women. Women Birth. 2012;25(3):e37-43. doi: 10.1016/j.wombi.2011.09.002. [PubMed: 21959041].

12. Gourounti K, Anagnostopoulos F, Lykeridou K, Griva F, Vaslamatzis G. Prevalence of women's worries, anxiety, and depression during pregnancy in a public hospital setting in Greece. Clin Exp Obstet Gynecol. 2013;40(4):581-3. [PubMed: 24597262].

13. Huizink AC, Mulder EJ, Robles de Medina PG, Visser GH, Buitelaar IK. Is pregnancy anxiety a distinctive syndrome? Early Hum Dev.
2004;79(2):81-91. doi: 10.1016/j.earlhumdev.2004.04.014. [PubMed: 15324989].

14. Abdi F, Kazemi F, Ramezani Tehrani F, Roozbeh N. Protocol for systematic review and meta-analysis: Hop (Humulus lupulus L.) for menopausal vasomotor symptoms. BMJ Open. 2016;6(4). e010734. doi 10.1136/bmjopen-2015-010734. [PubMed: 27105715]. [PubMed Central: PMC4853989].

15. Alderdice F, Lynn F, Lobel M. A review and psychometric evaluation of pregnancy-specific stress measures. J Psychosom Obstet Gy naecol.2012;33(2):62-77. doi:10.3109/0167482X.2012.673040. [PubMed: 22554138].

16. Terwee CB, Mokkink LB, Knol DL, Ostelo RW, Bouter LM, de Vet HC. Rating the methodological quality in systematic reviews of studies on measurement properties: A scoring system for the COSMIN checklist. Qual Life Res. 2012;21(4):651-7. doi: 10.1007/s11136-011-9960-1. [PubMed: 21732199]. [PubMed Central: PMC3323819].

17. Van den Bergh B. The influence of maternal emotions during pregnancy on fetal and neonatal behavior. Prenat Perinat Psychol J. 1990;5(2):119-30.

18. Huizink AC, de Medina PG, Mulder EJ, Visser GH, Buitelaar JK. Psychological measures of prenatal stress as predictors of infant temperament. JAm Acad Child Adolesc Psychiatry. 2002;41(9):1078-85. [PubMed 12218429].

19. Cote-Arsenault DY. Tasks of pregnancy and anxiety in pregnancy after perinatal loss: A descriptive study. University of Rochester; 1995. [Dissertation].

20. Rini CK, Dunkel-Schetter C, Wadhwa PD, Sandman CA. Psychological adaptation and birth outcomes: The role of personal resources, stress, and sociocultural context in pregnancy. Health Psychol.1999;18(4):333-45. [PubMed:10431934].

21. Orr ST, James SA, Casper R. Psychosocial stressors and low birth weight: Development of a questionnaire. I Dev Behav Pediatr. 1992;13(5):343-7. [PubMed: 1401118]

22. Levin JS. The factor structure of the pregnancy anxiety scale. J Health Soc Behav. 1991;32(4):368-81. [PubMed: 1765627]

23. Gloger-Tippelt G. A process model of the pregnancy course. Hum Dev. 1983;26(3):134-48. [PubMed: 6862429].

24. Roesch SC, Schetter CD, Woo G, Hobel CJ. Modeling the types and timing of stress in pregnancy. Anxiety Stress Coping. 2004;17(1):87-102. doi $10.1080 / 1061580031000123667$.

25. Meyer TJ, Miller ML, Metzger RL, Borkovec TD. Development and validation of the penn state worry questionnaire. Behav Res Ther 1990;28(6):487-95. doi: 10.1016/0005-7967(90)90135-6.

26. O'Connor TG, Winter MA, Hunn J, Carnahan J, Pressman EK, Glover V, et al. Prenatal maternal anxiety predicts reduced adaptive immunity in infants. Brain Behav Immun. 2013;32:21-8. doi 10.1016/j.bbi.2013.02.002. [PubMed: 23439080]. [PubMed Central: РMC3686987].

27. Pop VI, Pommer AM, Pop-Purceleanu M, Wijnen HA, Bergink V, Pouwer F. Development of the Tilburg pregnancy distress scale: The TPDS. BMC Pregnancy Childbirth. 2011;11:80. doi: 10.1186/1471-2393-11-80. [PubMed: 22029691]. [PubMed Central: PMC3216243]

28. Kornelsen J, Stoll K, Grzybowski S. Development and psychometric testing of the rural pregnancy experience scale (RPES). J Nurs Meas 2011;19(2):115-28. [PubMed: 22003812].

29. Spielberger CD. Manual for the state-trait anxiety inventory STAI (form $Y)$ ("self-evaluation questionnaire"). University at Buffalo Institutional Repository, New York: Center for Nursing Research; 1983.

30. Kazi A, Fatmi Z, Hatcher J, Niaz U, Aziz A. Development of a stress scale for pregnant women in the South Asian context: The A-Z stress scale. East Mediterr Health J. 2009;15(2):353-61. [PubMed: 19554982].

31. Green JM, Kafetsios K, Statham HE, Snowdon CM. Factor structure, validity and reliability of the Cambridge worry scale in a pregnant population. J Health Psychol. 2003;8(6):753-64. doi: 10.1177/13591053030086008. [PubMed:14670208] 
32. Carmona Monge FJ, Penacoba-Puente C, Marin Morales D, Carretero Abellan I. Factor structure, validity and reliability of the Spanish version of the Cambridge worry scale. Midwifery. 2012;28(1):112-9. doi: 10.1016/j.midw.2010.11.006. [PubMed: 21247673].

33. Goulet C, Polomeno V, Harel F. Canadian cross-cultural comparison of the high-risk pregnancy stress scale. Stress Med.1996;12(3):145-54. doi: 10.1002/(sici)1099-1700(199607)12:3<145::aid-smi686>3.0.co;2-e.

34. Redshaw M, Martin C, Rowe R, Hockley C. The Oxford worries about labour scale: Women's experience and measurement characteristics of a measure of maternal concern about labour and birth. Psychol Health Med. 2009;14(3):354-66. doi: 10.1080/13548500802707159. [PubMed: 19444713].

35. Wadhwa PD, Sandman CA, Porto M, Dunkel-Schetter C, Garite TJ. The association between prenatal stress and infant birth weight and gestational age at birth: A prospective investigation. Am J Obstet Gynecol. 1993;169(4):858-65. [PubMed: 8238139].

36. Theut SK, Pedersen FA, Zaslow MJ, Rabinovich BA. Pregnancy subsequent to perinatal loss: Parental anxiety and depression. J Am Acad Child Adolesc Psychiatry. 1988;27(3):289-92. doi: 10.1097/00004583198805000-00004. [PubMed: 3379012].

37. DiPietro JA, Hilton SC, Hawkins M, Costigan KA, Pressman EK. Maternal stress and affect influence fetal neurobehavioral development. Dev Psychol. 2002;38(5):659-68. [PubMed:12220045].

38. DiPietro JA, Christensen AL, Costigan KA. The pregnancy experience scale-brief version. JPsychosom Obstet Gynaecol. 2008;29(4):262-7. doi: 10.1080/01674820802546220. [PubMed: 19065395]. [PubMed Central: PMC2805904].

39. Da Costa D, Brender W, Larouche J. A prospective study of the impact of psychosocial and lifestyle variables on pregnancy complications. $J$ Psychosom Obstet Gynaecol.1998;19(1):28-37. [PubMed: 9575466].

40. Curry MA, Burton D, Fields J. The prenatal psychosocial profile: A research and clinical tool. Res Nurs Health. 1998;21(3):211-9. [PubMed: 9609506].

41. Yali AM, Lobel M. Coping and distress in pregnancy: An investigation of medically high risk women. J Psychosom Obstet Gynaecol. 1999;20(1):39-52. [PubMed:10212886].

42. Cha S, Masho SW. Preterm birth and stressful life events. In: Erez O, editor. Preterm birth. InTechOpen; 2013. doi: 10.5772/54978.

43. Nunnally JC, Bernstein IH. Validity. Psychometric theory. 3. New York: McGraw-Hill Book Company; 1994. p. 99-132.

44. Arch JJ. Pregnancy-specific anxiety: Which women are highest and what are the alcohol-related risks? Compr Psychiatry. 2013;54(3):21728. doi:10.1016/j.comppsych.2012.07.010. [PubMed: 22943960].

45. Lee AM, Lam SK, Sze Mun Lau SM, Chong CS, Chui HW, Fong DY. Prevalence, course, and risk factors for antenatal anxiety and depression. Obstet Gynecol. 2007;110(5):1102-12. doi: 10.1097/01.AOG.0000287065.59491.70. [PubMed: 17978126]. 